\title{
On the role of oxygen and glucose in bone marrow-derived mesenchymal stem cell proliferation
}

\author{
Sayed-Amir Marashi ${ }^{1}$ and Hamideh Fouladiha ${ }^{1}$ \\ ${ }^{1}$ Affiliation not available
}

January 31, 2022

Sayed-Amir Marashi*, Hamideh Fouladiha

Department of Biotechnology, College of Science, University of Tehran, Tehran, Iran.

E-mail address: marashi@ut.ac.ir (S.-A. Marashi)

\begin{tabular}{l} 
Abstract \\
Bone marrow mesenchymal stem cells (BM-MSCs) are one of the most popular cell types for tissue engineering and regener \\
\hline
\end{tabular}

\section{To the Editor:}

Mesenchymal stem cells (MSCs) are stromal cells that were originally reported in the bone marrow (BM) but can be isolated from various other tissues. MCSs can differentiate into multiple mesodermal cell lineages $[1]$.

BM-MSCs are used in numerous clinical applications including regenerative medicine [2]. Since MSCs are a rare cell population in the bone marrow, one should expand these cells for clinical applications. It is generally believed that reduced oxygen tension byin vivo transplantation plays a critical role in the success of implantation, and more specifically, cell survival and expansion [3]. However, BM-MSCs are shown to have excellent expansion when grown in hypoxic conditions [4]. This observation, indeed, questions the common belief on the importance of oxygen treatment for BM-MSC proliferation $[5,6]$.

To check whether oxygen or glucose supplementation is the most important factor in BM-MSC expansion, we used our previously published context-specific metabolic network model of these cells [7]. At three different oxygen-uptake rates, which can represent normoxia, physoxia and hypoxia, we computed cell growth rate as a function of glucose uptake rate.

To be specific, we used flux balance analysis (FBA) approach to simulate the cell growth at different oxygen and glucose consumption rates $[8,9]$. To this end, we assumed that all nutrients are available in excess, which, in our constraint-based model, translates to unbound uptake fluxes. Then, at three different oxygen-uptake flux values (i.e. , 0.1, 4, and 24), we used FBA to compute the maximum possible biomass production rate as a function of glucose uptake rate. In FBA-based simulations of metabolism, biomass production rate is typically considered as the objective function for modeling cell expansion and proliferation.

The results of our analysis are summarized in Figure 1. In this plot, Interestingly, one can observe that the three simulations result in almost similar growth patterns. Therefore, our model suggests that oxygen has only a minor influence on the proliferation of BM-MSCs. On the other hand, glucose uptake rate considerably changes the growth rate. On the other hand, the glucose uptake rate considerably changes the growth rate, which is in line with the recently published observations [8]. 
Here, we used a constraint-based model of BM-MSCs to investigate the effect of oxygen and glucose on the metabolism of these cells. Our results suggest that for cell expansion, glucose availability is more important compared to oxygen availability. In our simulations, possible oxygen-dependent gene regulation is ignored, i.e. , the same metabolic network model is used at all oxygen uptake rates. However, based on the recent observation that protein expression in MSCs is not affected by oxygen decrease, this assumption is acceptable [4]. One should note that, to a great extent, BM-MSCs rely on glycolytic metabolism, which limits the effect of oxygen. Consequently, availability of glucose to these cells seems to be a critically important factor.

Many tissue engineering studies have considered oxygen-releasing scaffolds for improving cell survival and proliferation postimplantation [9]. We want to emphasize here that for a successful tissue engineering practice, glucose-releasing scaffolds might also be a neglected necessity.

\section{References:}

[1] Ullah I, et al . Human mesenchymal stem cells-current trends and future prospective. Biosci Rep 2015;35:e00191.

[2] Lee SH. The advantages and limitations of mesenchymal stem cells in clinical application for treating human diseases. Osteoporos Sarcopenia 2018;4:150.

[3] Ferro F, et al . survival/adaptation of bone marrow-derived mesenchymal stem cells after long-term starvation through selective processes. Stem Cells 2019;37:813.

[4] Henrionnet C, et al . Hypoxia for Mesenchymal Stem Cell Expansion and Differentiation: The best way for enhancing TGF $\beta$-induced chondrogenesis and preventing calcifications in alginate beads. Tissue Eng Part A 2017;23:913.

[5] Gala DN, Fabian Z. To Breathe or Not to Breathe: The role of oxygen in bone marrow-derived mesenchymal stromal cell senescence. Stem Cells Int 2021;2021:8899756.

[6] Buizer AT. Towards in vivo application of oxygen-releasing microspheres for enhancing bone regeneration. Ph.D. Dissertation, University of Groningen, 2018.

[7] Fouladiha $\mathrm{H}$, et al . Reconstruction and validation of a constraint-based metabolic network model for bone marrow-derived mesenchymal stem cells. Cell Prolif 2015;48:475.

[8] Fouladiha H, Marashi SA. Biomedical applications of cell- and tissue-specific metabolic network models. J Biomed Inform 2017;68:35.

[9] Orth JD, et al. What is flux balance analysis? Nat Biotechnol 2010;28:245.

[10] Lau F, et al. Effects of oxygen and glucose on bone marrow mesenchymal stem cell culture. Adv Biosyst 2020;4:e2000094.

[11] Abudula T, et al. Oxygen-releasing antibacterial nanofibrous scaffolds for tissue engineering applications. Polymers 2020;12:1233. 


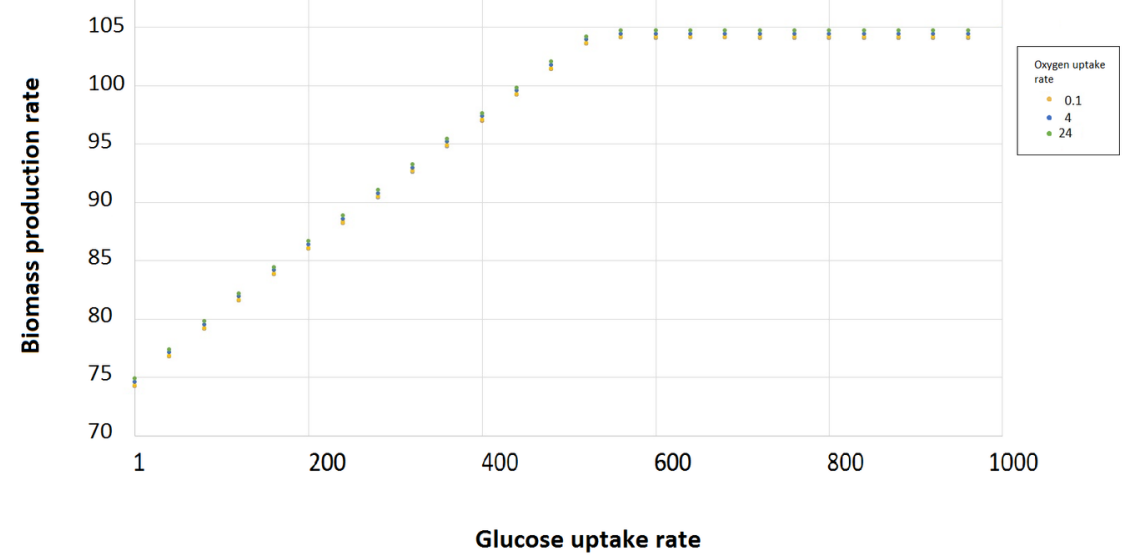

Figure 1. Biomass production rate of BM-MSCs as a function of glucose uptake rate. The growth simulations are performed using flux balance analysis method and iMSC1255 as the metabolic model [1]. Simulations are repeated at three different oxygen fluxes. 